\title{
Lesão da artéria ilíaca interna bilateral associada com trauma pélvico, tratada por intervenção arteriografia com embolização
}

\author{
Bilateral internal iliac artery injury associated \\ with pelvic trauma, treated with radiographic \\ intervention and embolization
}

\author{
Tiago Santoro Bezerra', Diogo Garcia², Francisco S. Collet e \\ Silva $^{3}$, Renato Sergio Poggetti ${ }^{4}$, Belchor Fontes $^{5}$, Dario Birolini ${ }^{6}$
}

\begin{abstract}
Bezerra TS, Garcia D, Collet e Silva FS, Poggetti RS, Fontes B, Birolini D. Lesão da artéria ilíaca interna bilateral associada com trauma pélvico, tratada por intervenção arteriografia com embolização. Rev Med (São Paulo). 2007 abr.-jun.;86(2):106-11.

RESUMO: Ruptura traumática do anel pélvico resulta geralmente de impacto com grande dispêndio de energia levando à ruptura do complexo ósteo-ligamentar levando a hemorragia retroperitoneal oriunda do plexo venoso posterior da pelve e de superfícies ósseo-esponjosas, que juntamente com lesões associadas acarreta cerca de $15 \%$ a $25 \%$ de mortalidade nos pacientes com lesão do anel pélvico ${ }^{1}$. Em menos de 10\% dos casos ocorre sangramento de artérias conhecidas, e em menos de $1 \%$ é relatado sangramento de vasos ilíacos ou femorais $2,3,4$. O objetivo do presente estudo é relatar o caso de um paciente vítima de trauma contuso com fratura pélvica e instabilidade hemodinâmica persistente após fixação pélvica externa, tendose diagnosticado por arteriografia lesão artérial ilíaca interna bilateral, e tratado por radiologia inervencionista com embolização.
\end{abstract}

DESCRITORES: Pelve/lesões. Radiologia intervencionista. Embolização terapêutica. Artéria ilíaca/lesões. Fraturas fechadas.

\footnotetext{
* Trabalho realizado no Serviço de Cirurgia de Emergência da Divisão de Clínica Cirúrgica III (SCE, DCCIII) do Hospital das Clínicas da Faculdade de Medicina da Universidade de Sâo Paulo (HC, FMUSP).

1. Residente de Cirurgia Geral ( $2^{\circ}$ Ano), HC, FMUSP.

2. Residente de Cirurgia Geral (4ำ Ano), HC, FMUSP.

3. Medico Assistente DCCIII, HC, FMUSP.

4. Diretor, SCE, DCCIII, HC, FMUSP.

5. Supervisor médico, SCE, DCCIII, HC, FMUSP.

6. Professor, Disciplina de Cirurgia do Trauma, Departamento de Cirurgia, FMUSP.

Endereço para correspondência: e-mail: tiagosbezerra@uol.com.br
} 


\section{INTRODUÇÃO}

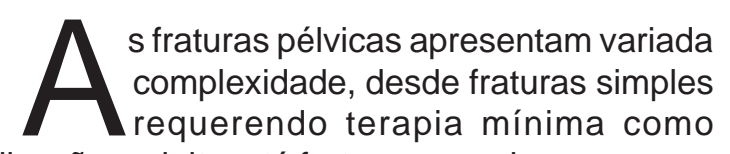
imobilização no leito, até fraturas complexas em que a fixação não é resolutiva, representando então um desafio ao cirurgião do trauma, requerendo a utilização de métodos diagnósticos/terapêuticos complementares como a radiologia intervencionista.

Embora a pelve óssea humana seja uma estrutura sólida, os traumatismos graves com grande impacto podem envolver dispêndio de energia suficiente para a ruptura do anel pélvico, geralmente com lesões associadas e grande hemorragia retroperitoneal, com índices de mortalidade de cerca de $15 \%$ a $25 \% 1$. Os quadros complexos com grande hemorragia usualmente resultam da ruptura do complexo ósteo-ligamentar, decorrente de fratura e/ ou luxação sacro-ilíaca ou de fratura sacral, sendo que a abertura do anel pélvico esgarça os plexos venosos pélvicos e ocasionalmente rompe o sistema arterial ilíaco interno. Geralmente a hemorragia decorre de lesão do plexo venoso posterior da pelve, e sangramento das superfícies ósseo esponjosas. Em menos de $10 \%$ dos casos, pode decorrer de sangramento de uma artéria conhecida, sendo que o sangramento de uma grande artéria é menos freqüente, e a literatura refere como menores que $1 \%$ os índices de sangramento dos vasos ilíacos ou femorais ${ }^{1,2,3}$.

As fraturas pélvicas com instabilidade hemodinâmica e do anel pélvico requerem, a par da reanimação hemodinâmica, estabilização do anel pélvico.

A estabilização do anel pélvico permite a mobilização precoce do paciente e diminui o índice de mortalidade. Inúmeros dispositivos como o fixador externo anterior, o fixador pélvico e as calças antichoque promovem rápida estabilização em situações de emergência, controlando a instabilidade hemodinâmica, mas não são o tratamento definitivo ideal, pois não controlam a instabilidade mecânica do anel pélvico posterior ${ }^{5,6,7}$. Na presença de uma ruptura instável do anel pélvico e um estudo abdominal positivo, a estabilização da pelve deve ser realizada antes da cirurgia. Se a estabilidade hemodinâmica não é obtida após colocação do fixador externo, pode ser realizada uma angiografia diagnóstica e terapêutica ${ }^{1,5}$.

O objetivo do presente estudo é relatar o caso de um paciente vítima de trauma contuso com fratura pélvica e instabilidade hemodinâmica persistente após fixação pélvica externa, tendo-se diagnosticado por tomografia lesão arterial ilíaca interna bilateral, e tratado por radiologia inervencionista com embolização.

\section{RELATO DO CASO}

Paciente masculino, R.S.M., 9anos, vítima de atropelamento por ônibus (roda parou em cima da bacia), trazido pelo resgate aéreo (helicóptero) e admitido cerca de 40 minutos após o acidente. Os dados locais informados pelos bombeiros indicaram: $\mathrm{FC}=160 \mathrm{bpm}$ (batimentos/minuto), pressão arterial não aferida, perfusão lentificada, FR: 36 incursões por minuto (ipm). GCS 14, administrados $100 \mathrm{~mL}$ de solução fisiológica (SF) ( $\mathrm{NaCl}$ a $0,9 \%)$ durante o transporte.A avaliação inicial à admissão do paciente revelou: A: Vias aéreas pérvias, ventilação espontânea, paciente com colar cervical, saturação 97\% com máscara; B: murmúrio vesicular audível bilateralmente, freqüência respiratória de $40 \mathrm{ipm}$; C: Bulhas cardíacas normofonédicas em dois tempos sem sopros. Pressão arterial inaudível, FC 170 bpm, abdômen doloroso difusamente. Pelve instável. Pulsos finos, perfusão lentificada, e hematúria após sondagem vesical. Iniciada reposição volêmica com $500 \mathrm{~mL}$ de SF, e solicitado sangue tipo específico; D: Glasgow 14, pupilas isocórica e fotorreagentes. $\mathrm{O}$ paciente movimentava os quatro membros; E: extenso ferimento descolante de coxa esquerda e dorso (Figura 1) e fratura pélvica (instabilidade nos componentes rotacional e vertical). Realizou-se exame radiológico do tórax que mostrou ausência de alterações, e da pelve que revelou fratura complexa com disjunção sínfise púbica e sacrilíaca bilateral (Figura 2). Os escores de gravidade do trauma determinados para o paciente foram: RTS 4,62, ISS 25 , TRISS $76,8 \%$. Frente ao quadro de instabilidade hemodinâmica, mecanismo de trauma e dor abdominal difusa foi indicada uma laparotomia exploradora de urgência e posterior fixação externa da pelve (Figura 3), que foram realizadas no centro cirúrgico. A laparotomia identificou um hematoma em zona III retroperitoneal, o qual não foi explorado cirurgicamente, e ausência de lesões detectáveis em outros órgãos.Foi realizada uma uma colostomia em alça de proteção no ângulo hepático do colón, tendo em vista 0 grande ferimento descolante comprometendo a coxa esquerda e o períneo. Foi realizada também a lavagem do cólon distal, e o tamponamento do ferimento descolante com compressas para controle do intenso sangramento venoso persistente. Durante a permanência na sala operatória o paciente recebeu $3500 \mathrm{ml}$ de cristalóide, $500 \mathrm{ml}$ de colóide e duas unidades de hemácias. 


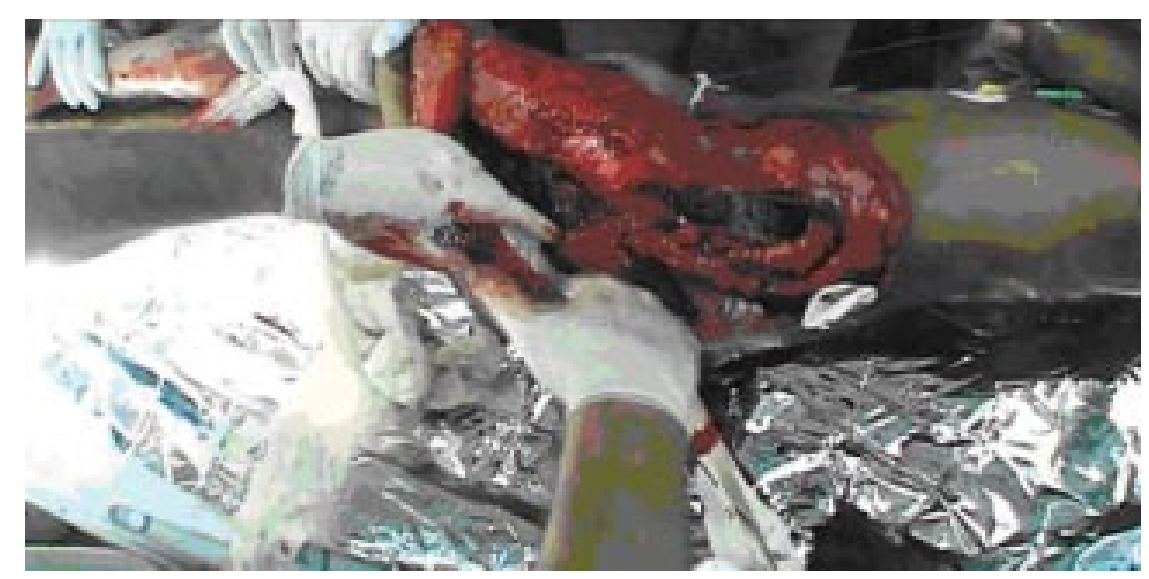

Figura 1. Descolante.

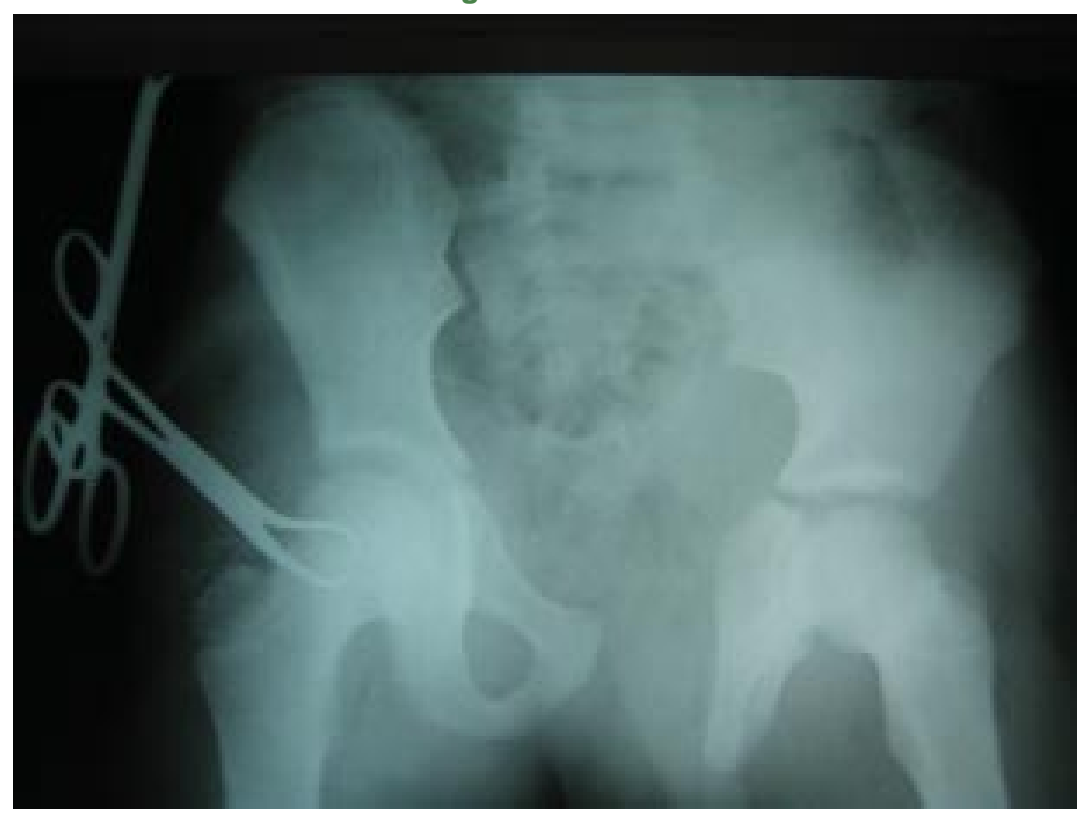

Figura 2. Fratura complexa com disjunção sínfise púbica e sacroilíaca bilateral.

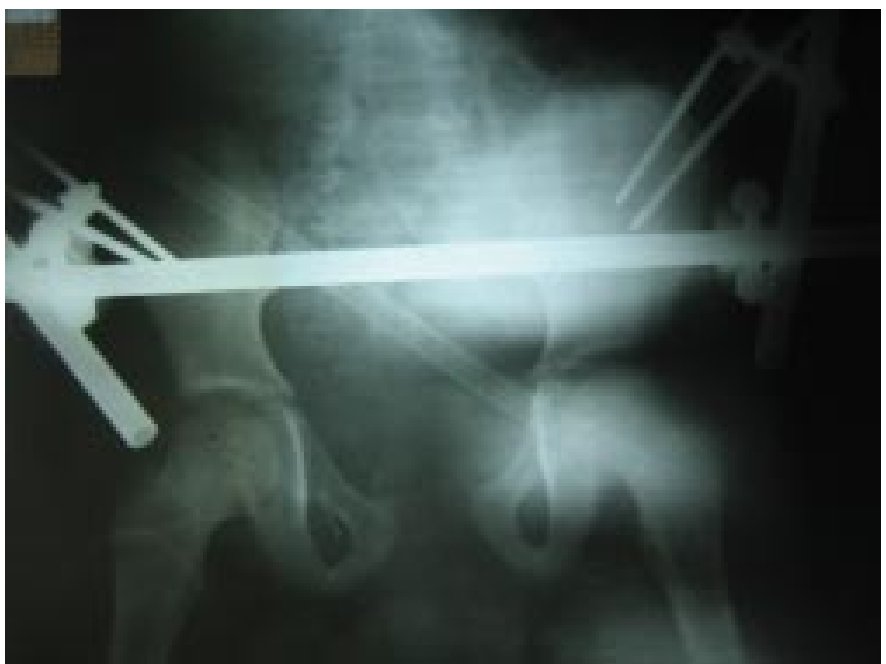

Figura 3. Fixador externo.

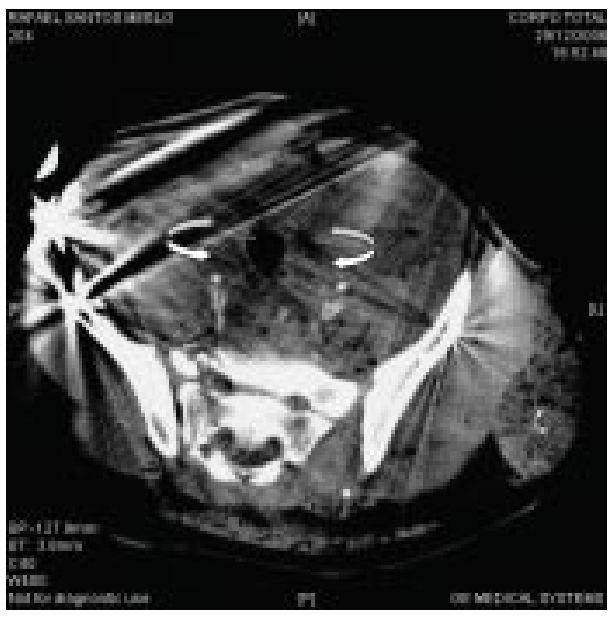

Figura 4. Sangramento ativo arterial pélvico bilateral, de ramos das artérias ilíacas internas. 
Após cirurgia paciente foi submetido a uma tomografia computadorizada de corpo inteiro que demonstrou moderado hemoretroperitonio e sinais de persistentes sangramento ativo arterial pélvico bilateral, proveniente de ramos das artérias ilíacas internas (Figura 4). O paciente foi então levado ao departamento de radiologia intervencionista para a realização de uma arteriografia que evidenciou sangramento ativo de ramo distal de artéria pudenda interna D (Figuras $5 \mathrm{a}$ e b) e sangramento ativo de artéria ilíaca interna $\mathrm{E}$ (Figura $5 \mathrm{c}$ ). Realizou-se então a embolização das artérias ilíacas internas bilaterais com gelfoam e molas (Figuras $5 \mathrm{~d}$ ). Durante a permanencia na arteriografia o paciente recebeu $1000 \mathrm{ml}$ de cristalóide, 2 unidades de hemácias e 2 unidades de plasma. Após este procedimento, o paciente foi levado para unidade de terapia intensiva onde apresentou melhora das condições clinicas, permanecendo estável no segundo dias pósembolização sem necessidade de drogas vasoativas e foi extubado no 9 PO. Foram realizadas quatro novas abordagens cirúrgicas para limpeza e desbridamento do ferimento descolante, tendo o paciente evoluído com boa granulação local e realizado enxerto com malha no $10 \mathrm{PO}$. Recebeu alta da unidade de terapia intensiva no $21^{\circ}$ dia pos trauma e alta hospitalar após 32 dias de internação. Atualmente está em acompanhamento ambulatorial após 4 meses do trauma, com boa recuperação da fratura de bacia, já sem o fixador externo, boa condição local do enxerto e aguardando cirurgia para reconstrução de transito intestinal.
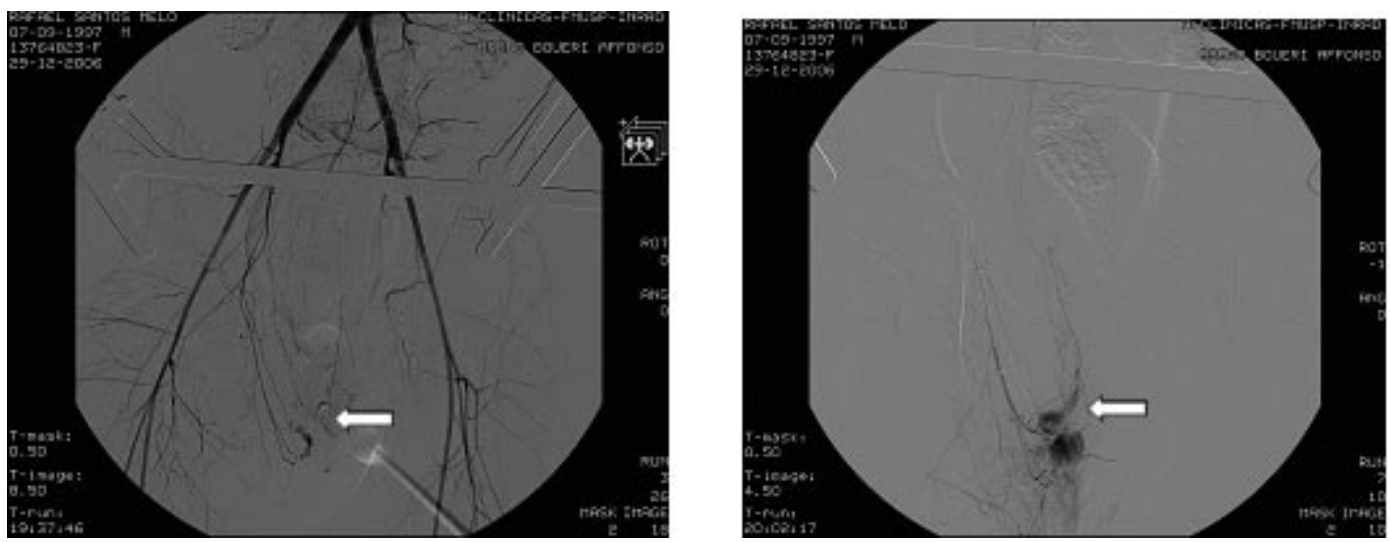

Figuras 5 a e b. Sangramento pudenda interna D.

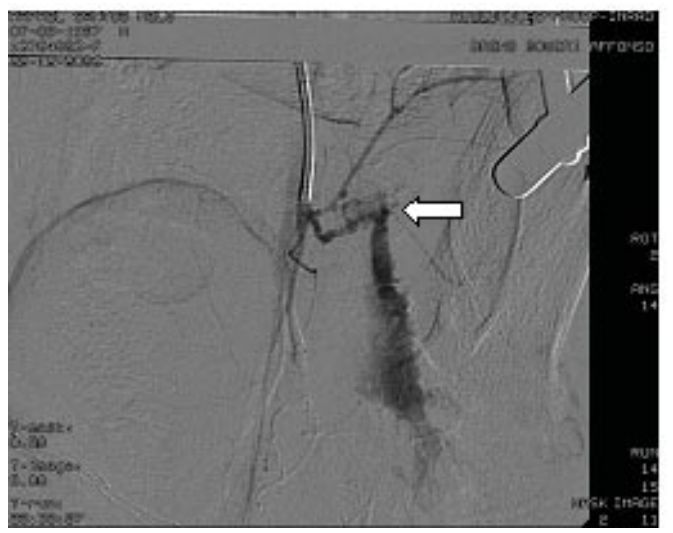

Figura 5c. Sangramento da art. llíaca interna D.

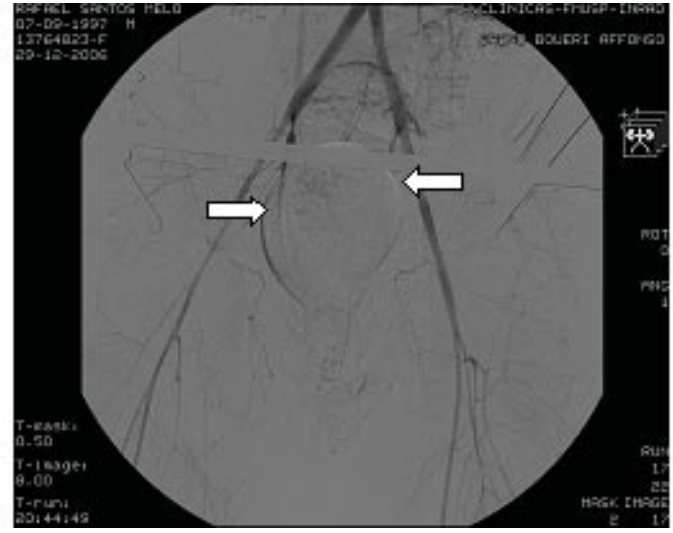

Figura $5 d$. Controle pós-embolização.

\section{DISCUSSÃO}

Todos os pacientes com trauma fechado, com impacto de alta energia, devem ser considerados como tendo possivelmente uma fratura pélvica. $O$ método padrão para o controle das hemorragias pélvicas é a aplicação de um fixador externo

anterior ${ }^{1,5,7}$. Grandes séries demonstram que a incidência de hemorragia arterial tratável através da embolização é de aproximadamente $10 \%$, portanto o uso destas técnicas deve ser reservado para os casos nos quais todos os métodos de controle da 
hemorragia já foram tentados, e nessas circunstância a embolização pode ser usada com sucesso ${ }^{1,2,3}$

No caso aqui relatado tal fratura foi diagnosticada ao exame físico do paciente. A diferenciação entre sangramento intra-abdominal e sangramento de uma fratura pélvica pode ser difícil. $\mathrm{O}$ paciente instável deve ser investigado para sangramento intra-abdminal, por meio da ultrasonografia (FAST) e/ou da lavagem peritoneal diagnóstico ${ }^{1,5}$. Caso o resultado seja positivo para hemoperitônio, o paciente deve ser submetido à laparotomia. Caso seja negativo e o paciente esteja estável, deve ser tratado de acordo com o tipo de fratura. No paciente citado tal investigação diagnóstica não foi possível, dada a urgência resultante da instabilidade hemodinâmica e dor abdominal difusa que justificaram a realização da laparotomia, permitindo identificar o hematoma retroperitoneal mas não a fonte do sangramento. Em pacientes traumati-zados graves com fratura pélvica, mesmo com indicação cirúrgica por hemoperitônio diagnosticado, mas com estabilidade hemodinâmica, pode ser feita uma tomografia computadorizada (TC) que poderá identificar tanto lesão retroperitoneal, quanto sangramentos arteriais, e/ou lesões abdominais associadas ${ }^{1,5,8}$. No caso deste estudo, após a laparotomia foi realizada a TC que concluiu a investigação diagnóstica, ao identificar sangramento pélvico bilateral persistente. A arteriografia tem indicação principalmente quando a hemorragia persiste após a redução da fratura pélvica, e define com precisão os sítios de sangramento. Tem-se observado que, com freqüência progressiva, tem permitido a realização de embolização terapêutica ${ }^{1,9,10,11}$. No caso aqui relatado, a arteriografia permitiu a identificação precisa dos sítios de sangramento em ramos diretos de cada artéria ilíaca interna, bem como a intervenção terapêutica definitiva por embolização dos vasos sangrantes.

\section{CONCLUSÃO}

O presente relato de caso ilustra o atendimento de um paciente traumatizado grave, apresentando instabilidade hemodinâmica persistente que requereu indicação de laparotomia de urgência, sem tempo para diagnósticos por imagem além da radiografia simples do tronco, que apenas confirmou a fratura pélvica. A laparotomia evidenciou o hematoma retroperitoneal mas não a fonte do sangramento, o qual persistiu e justificou a indicação da tomografia que evidenciou o sangramento pélvico bilateral persistente. Seguiu-se a realização da arteriografia que identificou com precisão lesão em ramo direto de cada artéria ilíaca interna, e permitiu o tratamento definitivo por embolização.

Bezerra TS, Garcia D, Collet e Silva FS, Poggetti RS, Fontes B, Birolini D. Bilateral internal iliac artery injury associated with pelvic trauma, treated with radiographic intervention and embolization. Rev Med (São Paulo). 2007 abr.-jun.;86(2):106-11.

\begin{abstract}
Pelvic ring ruptures are a cause of great mortality and morbidity in trauma patients. The fatalities are, in general, consequence of the retroperitoneal hemorrhage and other associated damages due to the great force necessary to rip the pelvic ring. The mortality in theses cases are between 15 and $25 \%$. The hemorrhage is usually due to the posterior pelvis venous plexus and the bony surface sponge bleedings. Less than $10 \%$ of the cases its caused by the bleeding of a known artery and in only $1 \%$. by iliac or femoral arteries The objectives of this study is to report a case of a patient victim of a blunt trauma, with a complex pelvic fracture and hypotension by a bilateral iliac artery injury that was diagnosed and treated by interventionist radiology.
\end{abstract}

KEY WORDS: Pelvis/injuries. Radiology, interventional. Embolization, therapeutic. Iliac artery/ injuries. Fractures, closed.

\title{
REFERÊNCIAS
}

1. Townsend CM, Beauchamp RD, Evers BM, Mattox KL. Sabiston tratado de cirurgia a base biológica da prática cirúrgica moderna. São Paulo: Elsevier; 2005.

2. Bem-Menachem Y. Exploratory angiography and transcatheter embolization for control of arterial hemorrhage in patients with pelvic ring disruption. Tech Orthop. 1995;9:271-4.
3. Buckle R, Browner B. Emergency reduction for pelvic ring disruptions and control of associated hemorrhage using the pelvic stabilizer. Tech Orthop. 1995;9:25866.

4. Klein S, Saroyan M, Baungartner F. Management strategy of vascular injures associated with pelvic fractures. J Cardiovasc Surg. 1992;33:349-57. 
5. Moore EE, Mattox KL, Feliciano DV. Manual do trauma. São Paulo: Artmed; 2006.

6. Martins HS, Damasceno MCT, Awada SB. Prontosocorro condutas do Hospital das Clínicas da Faculdade de Medicina da Universidade de São Paulo. São Paulo: Manole; 2007.

7. Cohen MT, Guimarães JM, Motta GRF. Fixação percutanea com parafuso iliossacral na lesão traumática do anel pélvico. Rev Bras Ortop. 2005;40(1/ 2).

8. Brown CV, Kasotakis G, Wilcox A, Rhee P. Demetriades SA. Does pelvic hematoma on admission computed tomography predict active bleeding at angiography for pelvic fracture? Am Surg. 2005;71(9):759-62.

9. Goslings JC, Van Delden OM. Angiography and embolisation to control bleeding after blunt injury to the abdomen or pelvis. Ned Tijdschr Geneeskd. 2007;151(6):345-52.

10. Van Vugt $A B$, Van Kampen $A$. An unstable pelvic ring. The killing fracture. J Bone Joint Surg Br. 2006;88(4):427-33.

11. Wild $M$, Weigand $H$, Steingässer $C$, Wenda $K$. Angiographic embolization of seriously bleeding vessels as an emergency procedure after traumatic disruption of the symphysis. Zentralbl Chir. 2005;130(2):170-3. 